\title{
Sporothrix schenckii endophthalmitis presenting as granulomatous uveitis
}

\author{
Mont J Cartwright, Mark Promersberger, Garth A Stevens
}

\begin{abstract}
Sporothrix schenckii occurs worldwide as a chronic subcutaneous mycotic infection, but can result in intraocular infection of cryptogenic origin without evident systemic infection. A case of $S$ schenckii endophthalmitis is presented without a history of trauma or systemic infection originally diagnosed as granulomatous uveitis that resulted in scleral perforation.

(Brf Ophthalmol 1993; 77: 61-62)
\end{abstract}

\section{Case report}

A 24-year-old black male with no prior ocular history and 20/20 vision was diagnosed with asymptomatic sarcoid by biopsy at bronchoscopy after a routine chest $x$ ray demonstrated hilar adenopathy. Fungal stains of the lung specimen were negative. Eighteen months later he presented with anterior granulomatous uveitis of the right eye. He was treated with cycloplegics, topical oral, and periocular steroids. Persistent inflammation led to seclusion pupillae with elevated intraocular pressure 1 month later. After a failed laser iridotomy a supernasal sector iridectomy was performed. Postoperatively he developed severe intraocular inflammation and dense cataract. One month after sector iridectomy he underwent cataract removal by aspiration. Postoperatively, he received two additional steroid injections at 1 month intervals in the inferotemporal and superotemporal sub-Tenon spaces for persistent inflammation. Best postoperation vision was 20/400 secondary to persistent uveitis.

Two weeks after cataract surgery, a white avascular elevation of perilimbal conjunctiva at 9 o'clock was noted. This was interpreted as necrotic tissue secondary to surgery. Five weeks after cataract surgery he complained of a sudden decrease in vision in the right eye. Visual acuity

University of Michigan, WK Kellogg Eye Center, Ann Arbor, MI 48105,

USA

M J Cartwright

Ophthalmology

Department, Medical

College of Virginia,

Richmond, Virginia, USA

$M$ Promersberger

$G$ A Stevens

Ophthalmology Section, McGuire VA Medical Center, Richmond, Virginia, USA

G A Stevens

Correspondence to: Mont J Cartwright, MD 24035 Piragua Place, Laguna, Niguel, CA 92677, USA.

Accepted for publication 17 September 1992 was hand motions; the eye was hypotonic with a

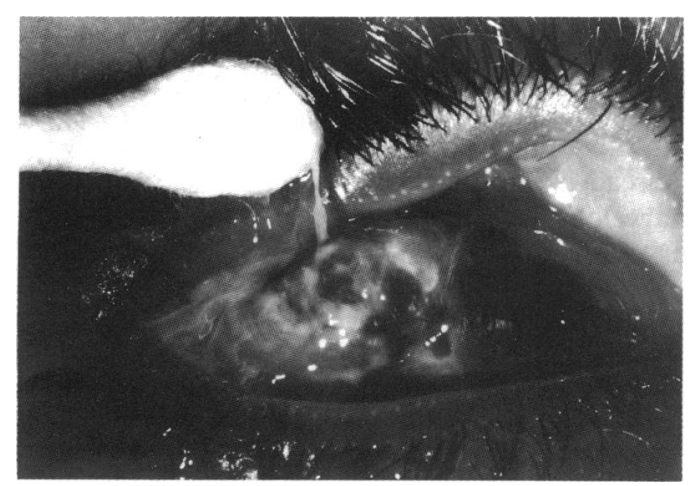

Figure 1 Right eye. Necrotic bulging temporal sclera with extruding vitreous on cotton swab.

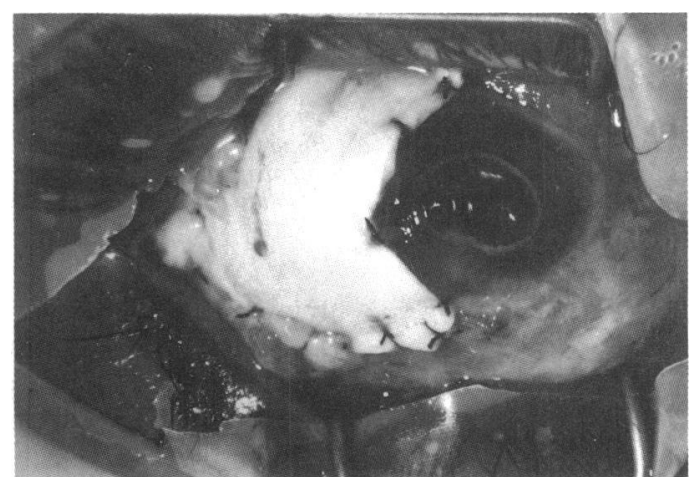

Figure 2 Right eye. Immediate postoperative photograph showing sutured homologous scleral patch in position.

grey-blue bulging of uveal tissue through necrotic sclera at the 9 o'clock position (Fig 1). Vitreous was extruding through a small perforation site. The scleral perforation was located posterior and inferior to the cataract incision and inferior to the depot steroid injection sites. Debridement of the necrotic tissue, cultures, and a scleral patch graft was performed (Fig 2). Intraoperatively, he received intravitreal injections of gentamicin, vancomycin, and clindamycin, and he was discharged on oral cephradine and bacitracin ointment. Initial cultures were negative. One week postoperatively, the eye was soft and vision was light perception. Cultures of the wound and vitreous were then positive for $S$ schenckii, later shown to be sensitive to amphotericin $\mathrm{B}$ and ketoconazole (Fig 3). Additional questioning of the patient

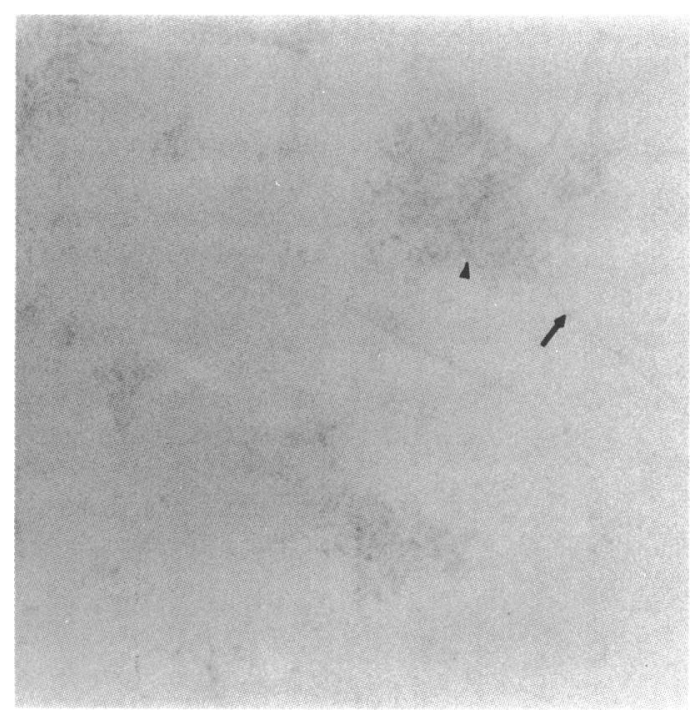

Figure 3 Right eye. Direct mount from vitreous culture of Sporothrix schenckii $\left(24^{\circ} \mathrm{C}\right.$ ). Piriform conidia (arrow head) adherent to hyphae (arrow). 


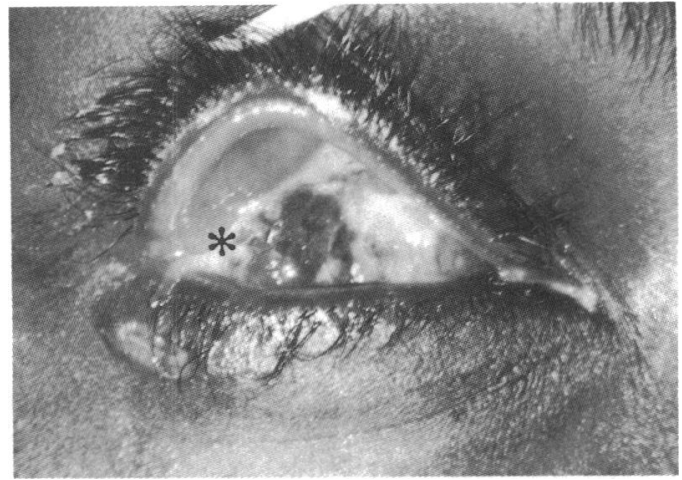

Figure 4 Right eye. Lower lid subciliary fungal abscess inferomedial to the lateral canthus (arrow). Note partial dissolution of the scleral patch graft $\left(^{\star}\right)$.

revealed no history of ocular trauma; however, he did a substantial amount of work using a power mower. $\mathrm{He}$ received an intravitreal injection of $5 \mu \mathrm{g}(0.1 \mathrm{ml})$ amphotericin $\mathrm{B}$, daily subconjunctival injections of $750 \mu \mathrm{g}(0.375 \mathrm{ml})$ amphotericin $\mathrm{B}$, in addition to natamycin drops every hour. The patient developed a lower lid fungal abscess 2 weeks after initiation of local antifungal therapy (Fig 4) which resolved with oral potassium iodide. Despite therapy, the eye became phthisical and enucleation was advised but declined by the patient.

\section{Pathology}

Specimens from the sector iridectomy and the iridectomy with cataract surgery were examined by haematoxylin and eosin, glyceryl monostearate, and periodic acid Schiff stains. Both the original and subsequent iridectomy specimens demonstrated plasma cells, lymphocytes, and epithelioid cells. No foreign bodies of fungal elements were seen on the first specimen. The second iris specimens demonstrated, in addition to granulomatous inflammation, a birefringent particle within the substance of the iris, interpreted as talc, but no organisms were identified.

\section{Comment}

$S$ schenckii is a saprophytic, dimorphic organism that can be isolated from a variety of plants. The most common type of infection is the lymphocutaneous form. Initial lesions resemble warts, furuncles, or epithelioma that can culminate in indolent, indurated, inflamed subcutaneous nodules distributed along the course of the regional lymphatics. Pulmonary sporotrichosis results from the inhalation of conidia. Sporotrichosis may also present in a disseminated form owing to haematogenous spread from unidentified cutaneous or pulmonary lesions, and may manifest as arthritis, sinusitis, meningitis, and ocular infections.'
The histopathological diagnosis of $S$ schenckii is often difficult. In primary lesions, the free yeast forms are rarely found in tissue sections stained with haematoxylin and eosin and may not be identified despite stains with periodic acid Schiff or glyceryl monostearate, making recovery in culture the most accurate method of diagnosis.'

Intraocular infections may be due to direct extension from the lid or conjunctiva, but most are endogenous, owing to systemic infection, and usually lead to loss of the eye. Ocular (and other monofocal sites) infections, without an antecedent history of trauma, or evidence of systemic disease are rare, but may be more common with $S$ schenckii than with other causes of fungal endophthalmitis. ${ }^{2}$ Latency in an animal model has been reported. ${ }^{3}$ Clinical manifestations include uveitis, scleral abscess, iridocyclitis, endophthalmitis, and retinochoroiditis. The clinical course is usually protracted, lasting for months.

Potassium iodide is an effective therapy and most patients will respond well to treatment, even in fairly advanced states. Amphotericin B and the imidazoles may be effective local and systemic therapy. Topical natamycin is an important ocular antifungal agent for filamentous organisms; however, reports suggest limited utility with this fungus. ${ }^{+}$

In this case, it is unclear at what stage $S$ schenckii became the primary pathogenic mechanism. Possible routes of infection include unreported trauma, iatrogenic infection associated with injections or surgery, or haematogenous spread from an undetected, asymptomatic, pulmonary infection. The occurrence of iatrogenic introduction, though reported in the literature, is unlikely as all procedures were performed with standard precautions. Unrecognised ocular trauma remains a remote possibility. More likely the original diagnosis of sarcoid may have been erroneous; though the lung biopsy was consistent with sarcoid, no active systemic disease was detected.

The refractory response of this unilateral anterior uveitis to topical and periocular steroids would suggest a primary diagnosis of cryptogenic endophthalmitis that eventually resulted in scleral perforation. This report emphasises that presumed non-infectious uveitis, not responsive to immunosuppressives, may be of an infectious aetiology.

1 Green WR. Ophthalmic pathology. Philadelphia: Saunders, 1986: vol 3, 1897-1905.

2 Font RL, Jakobiec FA. Granulomatous necrotizing retinochoroiditis caused by Sporothrix schenckii. Arch Ophthalmol 1976; 94:1513-9.

3 MacDonald E, Evert A, Reitmeyer JC. Reappearance of Sporothrix schenckii lesions after administration of Solu-nedid Sporothrix schenckii lesions after administration of
$\mathbf{R}$ to infected cats. Sabouraudia 1980; 18: $295-300$.

4 Johns K, O'Day D. Pharmacologic management of keratomycoses. Surv Ophthalmol 1988; 33: 178-88. 